\title{
LOFAR and Radio Loud AGN
}

\section{Wendy L. Williams ${ }^{1,2}$, Huub Röttgering ${ }^{1}$ and Reinout van Weeren ${ }^{3}$ on behalf of the LOFAR collaboration}

\author{
${ }^{1}$ Leiden Observatory, Leiden University, P.O. Box 9513, NL-2300 RA, Leiden, The Netherlands \\ ${ }^{2}$ Netherlands Institute for Radio Astronomy (ASTRON), \\ PO Box 2, 7990AA Dwingeloo, The Netherlands \\ ${ }^{3}$ Harvard-Smithsonian Center for Astrophysics, 60 Garden Street, Cambridge MA 02138, USA \\ email: wwilliams@strw.leidenuniv.nl
}

\begin{abstract}
At very low frequencies, the new pan-European radio telescope, LOFAR, is opening the last unexplored window of the electromagnetic spectrum for astrophysical studies. LOFAR will deeply survey the northern sky from $\sim 10$ up to $240 \mathrm{MHz}$. In this contribution we briefly describe some of the capabilities of LOFAR and the surveys planned to study fundamental issues related to the formation and evolution of galaxies and clusters of galaxies. We describe some of the challenges of low frequency observations with LOFAR and our progress in overcoming them. Further, we present some recent results from the ongoing imaging commissioning efforts. In the second part we discuss our studies of Low Excitation and High Excitation Radio Galaxies in the Boötes field and how LOFAR Surveys will help in studying their evolution.
\end{abstract}

Keywords. Galaxies: active, surveys, telescopes, Instrumentation: interferometers

\section{LOFAR}

LOFAR, the Low Frequency Radio Array, is a new low freqency radio telescope in the Netherlands and surrounding European countries. Its revolutionary design makes use of phased arrays instead of the traditional and expensive dishes. The simple receivers are turned into a real telescope electronically. While LOFAR has been described in detail elsewhere (see van Haarlem et al. 2013), we provide here a brief overview of the telescope.

The array is composed of several 'stations' each containing a number of simple dipoles. There are two types of dipole antennas, one for the Low Band Array (LBA) operating at $10-80 \mathrm{MHz}$ range and one for the High Band Array (HBA) operating in the $110-$ $240 \mathrm{MHz}$ range. As of Summer 2013, the Dutch part of the array consists of 37 stations; six stations reside in the $320 \mathrm{~m}$ diameter densely-packed 'Superterp', 24 are core stations located within a central $2 \mathrm{~km}$ radius, and the remaining 13 are remote stations going out to $80 \mathrm{~km}$. The array is extended to baselines of up to $1200 \mathrm{~km}$ with a further eight stations in a number of other European countries: Germany (5), the UK (1), Sweden (1), and France (1). The array status is shown in Fig. $1 \dagger$. It is very easy to add additional stations to the existing network as funds become available.

The signals from the antennas are digital, meaning that many beams or pointings can be formed simultaneously. This makes LOFAR an extremely efficient instrument for surveying large areas. The electronic nature of the beams makes LOFAR highly flexible. In terms of frequencies, up to 488 subbands of $195.312 \mathrm{kHz}$ or $156.250 \mathrm{kHz}$ are available, which can give a total bandwidth of up to $95 \mathrm{MHz}$. The Dutch array provides resolutions of $\sim 10^{\prime \prime} \times 40^{\prime \prime}$ at $60 \mathrm{MHz}$ and the inclusion of international baselines gives sub-arcsecond resolution in both the low and high bands $\left(\sim 0.7^{\prime \prime}\right.$ at $60 \mathrm{MHz}$ and $\sim 0.2^{\prime \prime}$ at $\left.240 \mathrm{MHz}\right)$.

$\dagger$ up-to-date information on the current status of LOFAR is available at http://www.astron.nl/ heald/lofarStatusMap.html, powered by Google Maps. 


\section{LOFAR Challenges and Progress}

Many functional elements of the LOFAR imaging system are already in place. Here we briefly mention some of the challenges within the imaging process that have either been solved or are currently being worked on:

Data rates of up to Tbs/s Advances in computer storage and power, combined with the high speed connections between the stations and the correlator in Groningen, allow us to handle the high data rates from LOFAR, although correlating and processing the data still requires supercomputers. Already, there are several peta-bytes of data stored in the long term archive (LTA).

Radio frequencies interference (RFI) Flagging of RFI is a crucial step in the data analysis. Despite the high levels of RFI in Northern Europe, the high frequency and time resolution of LOFAR observations combined with clever algorithms such as that of Offringa et al. (2010) allow for the rejection of RFI with typically $<10 \%$ loss to the data at the worst freqencies.

Removal of A-team sources LOFAR's LBA system has an extremely large field of view; the individual dipoles essentially "see" the entire sky. Even within the formed station beam, the few extremely bright radio sources at low frequencies (Cygnus A and Cassiopeia A and the other so-called "A-team") dominate any LBA observation for which they are above the horizon. Simply subtracting their contributions is time-consuming and computationally intensive. Instead we have been using, very successfully, the "demixing" method of van der Tol et al. (2007).

Beam calibration LOFAR has several beams: the dipole beams, the tile beams (for the HBA), the station beams and the interferometric beam. With the exception of the dipole beam, all are formed digitally and are both complex-valued and complex and time-variable. The theoretical beams are included in the LOFAR calibration software, and have been verified to be reasonably accurate through commissioning projects.

Wide-field mapmaking The wide field of view of LOFAR makes it necessary to implement wide-field imaging techniques such as $w$-projection. Moreover, the time-varying beams and ionoshperic corrections, as well as the direction-dependent ionosphere corrections required, mean it is necessary to implement the $A$-projection (Bhatnagar et al. 2008 ) in imaging. Both these algorithms have been combined in the LOFAR AWimager (Tasse et al. 2013).

Timing of the station clocks One of LOFAR's key advantages is that individual stations are cheap; however, they have cheap clocks which slowly drift. The station clocks can be calibrated by making use of the very wide frequency coverage and to simultaneously observe a calibrator source. This is however not trivial, and the details are still being worked on.

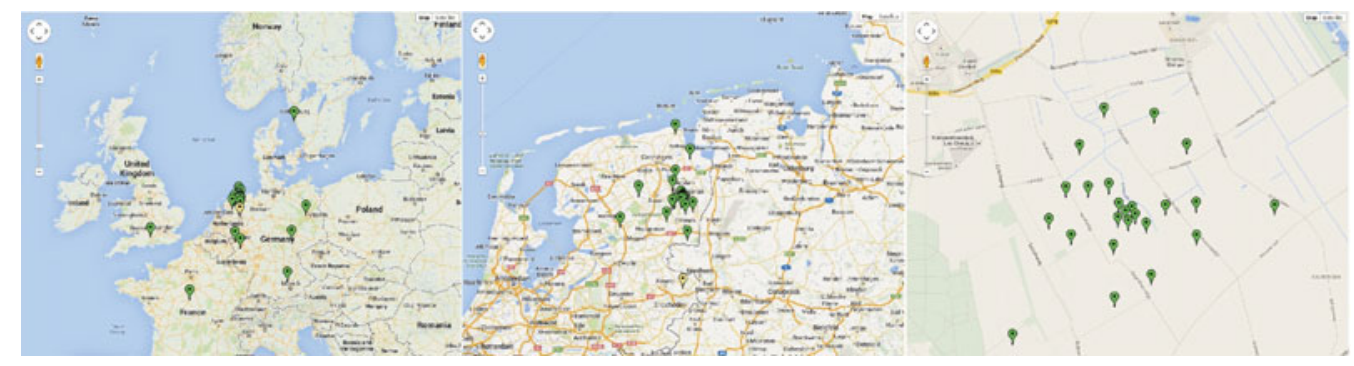

Figure 1. Current status of the LOFAR Array at three scales: left European scale showing the international stations; centre the Dutch scale showing the remote stations; and right the LOFAR core. The images are taken from George Heald's LOFAR Status map. 
Ionosphere As the sun becomes more active, it is increasingly more important to correct for the effects of ionospheric phase distortions in LOFAR observations. Similar to optical "seeing" the ionosphere shifts the positions of radio sources and in bad cases distorts their shapes. The shifts and distortions depend on the ionosphere in the direction of each source and so can vary strongly accross a single LOFAR field. Experience with ionospheric corrections at low freqencies with the VLA and GMRT led to the development of the SPAM algorithm (Intema et al. 2009) which is being implemented and further developed for LOFAR.

\section{LOFAR Science and Surveys}

The primary scientific drivers for LOFAR are encapsulated in the Key Science Projects: (i) Epoch of Reionisation; (ii) Surveys; (iii) Transients; (iv) Cosmic Rays; (v) Cosmic Magnetism; and (vi) Solar Science and Space Weather. The Surveys project is most relevant to these proceedings, which together with projects like Cosmic Magnetism, will provide images of nearby galaxies, AGN, clusters and large deep extragalactic fields.

The three fundamental areas of astrophysics that have driven the design of the planned LOFAR surveys are: (i) forming massive galaxies at the epoch of reionisation, (ii) magnetic fields and shocked hot gas associated with the first bound clusters of galaxies, and (iii) star formation processes in distant galaxies. To achieve the goals of the LOFAR surveys, a tiered approach will be used (for details see Röttgering et al. 2010): Tier-1 is an all sky survey at 15,30,60 and $120 \mathrm{MHz}$; Tier-2 is a medium deep survey over 1000 square degrees at 30,60, 120 and $200 \mathrm{MHz}$; and Tier-3 encompasses about 100 square degrees down to an extreme depth of $6 \mu \mathrm{Jy} \mathrm{rms}$ at $150 \mathrm{MHz}$. The areas, depths and frequencies of the surveys have been chosen so that they would contain: (i) 100 powerful radio galaxies close to or at the epoch of reionisation, (ii) 100 radio halos at the epoch when the first massive bound galaxy clusters appeared, and (iii) 100 proto-clusters. The depth versus frequency of the proposed surveys is given in Fig. 2. Another important

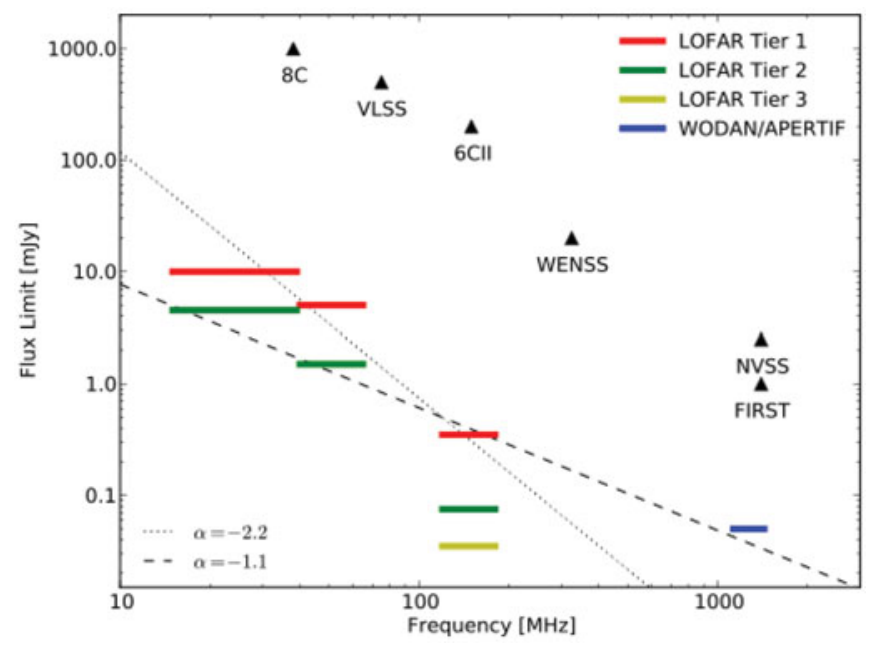

Figure 2. Flux limits $(5 \sigma)$ of the proposed LOFAR surveys compared to other existing radio surveys. The triangle represent existing surveys: NVSS, FIRST, WENSS, 6C, VLSS and 8C. The lines represent different power-laws $\left(S \sim \nu^{\alpha}\right.$ with $\alpha=-1.1$ and -2.2$)$ to illustrate how, depending on the spectral indices of the sources, the LOFAR surveys will compare to other current surveys. The proposed APERTIF/WODAN survey is also shown. 
motivation of LOFAR is to provide the entire international astronomical community with unique surveys of the radio sky that have a long-lasting legacy value for a broad range of astrophysical research. The fundamental astrophysical research topics for which LOFAR surveys will have a significant impact include (i) the formation and evolution of large scale structure of the Universe, (ii) the physics of the origin, evolution and end-stages of radio sources, (iii) the magnetic field and interstellar medium in nearby galaxies, and (iv) Galactic sources such as supernova remnants, HII regions, exoplanets and pulsars.

The LOFAR surveys will have excellent synergies with other proposed surveys, including the WODAN, EMU and optical surveys such as Euclid. WODAN (Westerbork Observations of the Deep APERTIF Northern-Sky) will be a deep (10 $\mu \mathrm{Jy})$ all-(Northern)-sky survey at $1400 \mathrm{MHz}$ using the phased array feeds to be installed on the Westerbork Radio Telescope (WSRT). EMU (Evolutionary Map of the Universe, see the contribution by Ray Norris in these proceedings), using the Australian SKA Pathfinder (ASKAP), will do the same for the Southern sky. Euclid is an ESA mission to be launched in $~ 2020$ and will survey 15, 000 square degrees (also a deep survey of 40 square degrees) providing visual images down to $\mathrm{AB}=24.5 \mathrm{mag}$; NIR images in $Y, J$ and $H$ bands down to $\mathrm{AB}=24$ mag; and NIR slitless spectroscopy. Primarily a Dark Energy mission, Euclid will provide shapes and photometric redshifts for $\sim 1.5$ billion galaxies.

\section{Recent LOFAR Results}

It should be noted that at present the measured noise in LOFAR images is dominated by calibration and imaging errors and a significant amount of both continued commissioning and technical research will be needed to obtain maps with the theoretical noise levels. However, the maps that are already being produced are the deepest ever at these low frequencies and high resolutions. The ongoing commissioning and early-science observations are producing some excellent imaging results. We highlight here a few recent results. For nearby galaxies, de Gasperin et al. (2012) have studied Virgo A (M87) in detail. This bright source is difficult to image because it is very bright and it lies at low declination for LOFAR. Nevertheless, they obtain very good images with noise levels of $\sim 6 \mathrm{mJy} /$ beam at $140 \mathrm{MHz}$ with $19^{\prime \prime} \times 14^{\prime \prime}$ resolution and $0.6 \mathrm{Jy} /$ beam at $25 \mathrm{MHz}$ with $85^{\prime \prime} \times 44^{\prime \prime}$ resolution. The Epoch of Reionisation group makes images with dynamic ranges of the order of $800,000: 1$, resolutions down to $15^{\prime \prime}$, noise levels of $100 \mu \mathrm{Jy} / \mathrm{beam}$ across a wide band of $120-180 \mathrm{MHz}$ (see Yatawatta et al. (2013) for some recent results).

Extragalactic fields that have been imaged with LOFAR include the Groth Strip (part of the calibrator, 3C295, field) and Boötes (see van Weeren et al. in prep) with images at $55-70 \mathrm{MHz}$ (noise $4-5 \mathrm{mJy}$, resolution $\left.20^{\prime \prime}\right), 40-50 \mathrm{MHz}\left(6-7 \mathrm{mJy}, 30^{\prime \prime}\right.$ ), and 30-40 MHz (11 mJy, 40"), revealing sources deeper than are visible in VLSS (at $74 \mathrm{MHz}$ ). Moreover, source counts have been determined for these fields. See also the contribution in these proceedings by Prandoni et al. where they present LOFAR observations of the Lockman Hole. Exploiting the very large field of view and looking at sources detected in the cluster field A2256 (see van Weeren et al. 2012), Rafferty et al. (in prep), have studied the low frequency SEDs of WISE-selected starbursts. They find that most $(\sim 75 \%)$ of the starbursts have SEDs that continue to rise below $\sim 60 \mathrm{MHz}$.

\section{LOFAR and AGN Studies}

AGN activity occurs in at least two different modes, each of which may be important, in different ways, for feedback on their host galaxies. The most commonly considered mode of AGN accretion is the 'standard' mode associated with quasars. This 


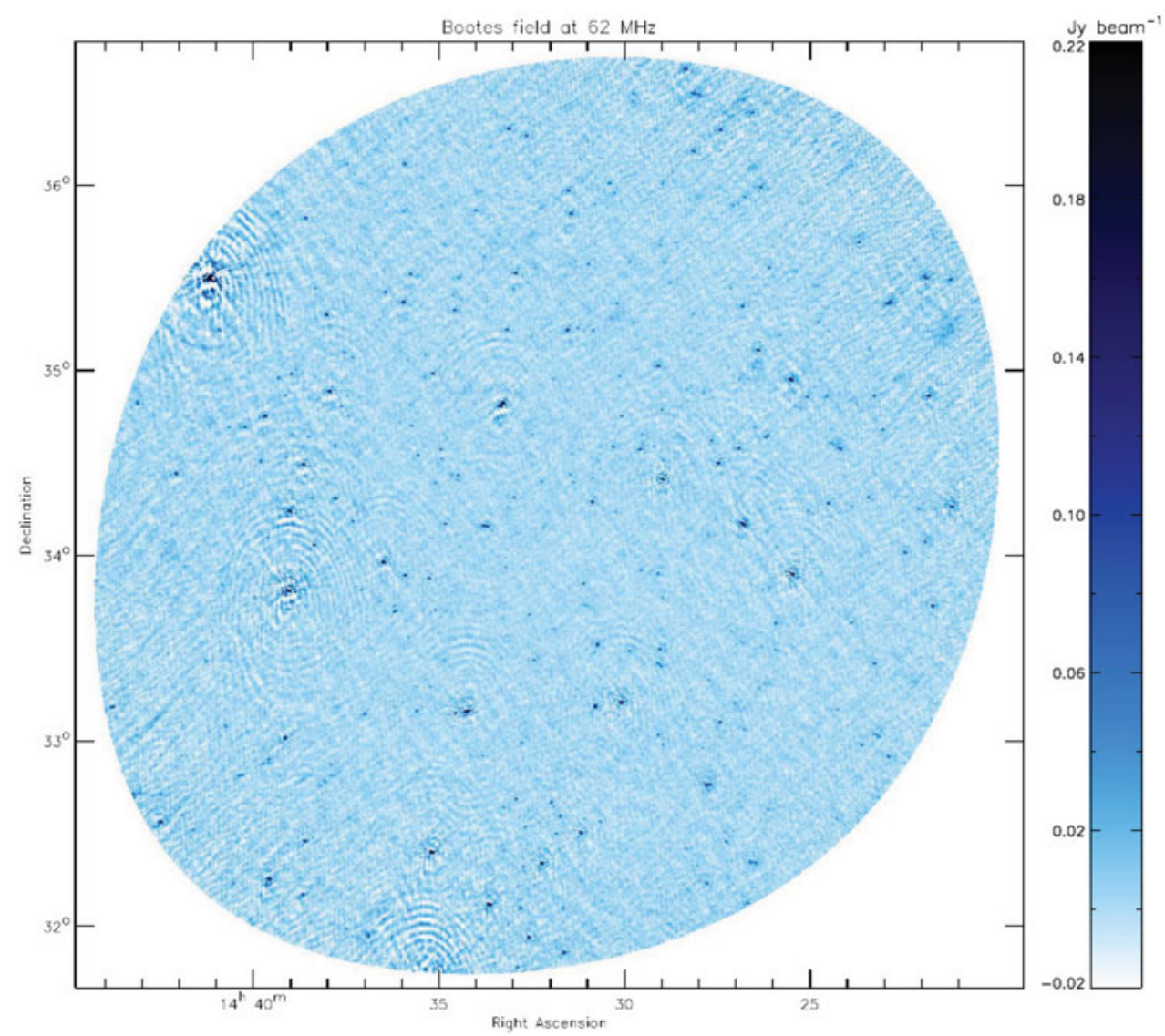

Figure 3. The Boötes field observed with LOFAR at $62 \mathrm{MHz}$ mosaic. The image covers 19 square degrees. The average noise is $4.8 \mathrm{mJy} \mathrm{beam}^{-1}$ and the resolution is $31^{\prime \prime} \times 19^{\prime \prime}$ (van Weeren et al. in prep).

mode, often and variously referred to as 'quasar-mode', 'cold-mode', 'radiative-mode', or 'high-excitation', is characterised by strong optical emission lines, MIR emission and/or absorption associated with a dusty torus (or other absorbing structure), along with possible X-ray emission. Here material is accreted on to the black hole through a radiatively efficient accretion disc. The second mode of AGN activity occurs with little radiated energy, but can lead to the production of highly energetic radio jets. Here, the strong emission lines are absent (Hine \& Longair (1979)) and they lack other evidence for accretion disks. These 'radiatively inefficient', 'radio-mode', 'hot-mode', or 'low-excitation' AGN are thought to be fueled by radiatively inefficient advection-dominated accretion flows (ADAFs) (e.g. Narayan \& Yi 1995).

Using the SDSS value-added spectroscopic sample of radio loud galaxies (Best \& Heckman 2012) which has been classified into Star-Forming and Low Excitation and High Excitation radio AGN, Janssen et al. (2013) looked at the fraction of galaxies that are hot-mode radio emitters as a function of their SDSS colours. LOFAR will be invaluable in extending samples such as these out to higher redshifts, and will shed light on the different evolution of the two accretion modes.

In anticipation of the LOFAR surveys we have surveyed the Boötes field with the GMRT at $153 \mathrm{MHz}$. The Boötes field is part of the NOAO Deep Wide Field Survey 


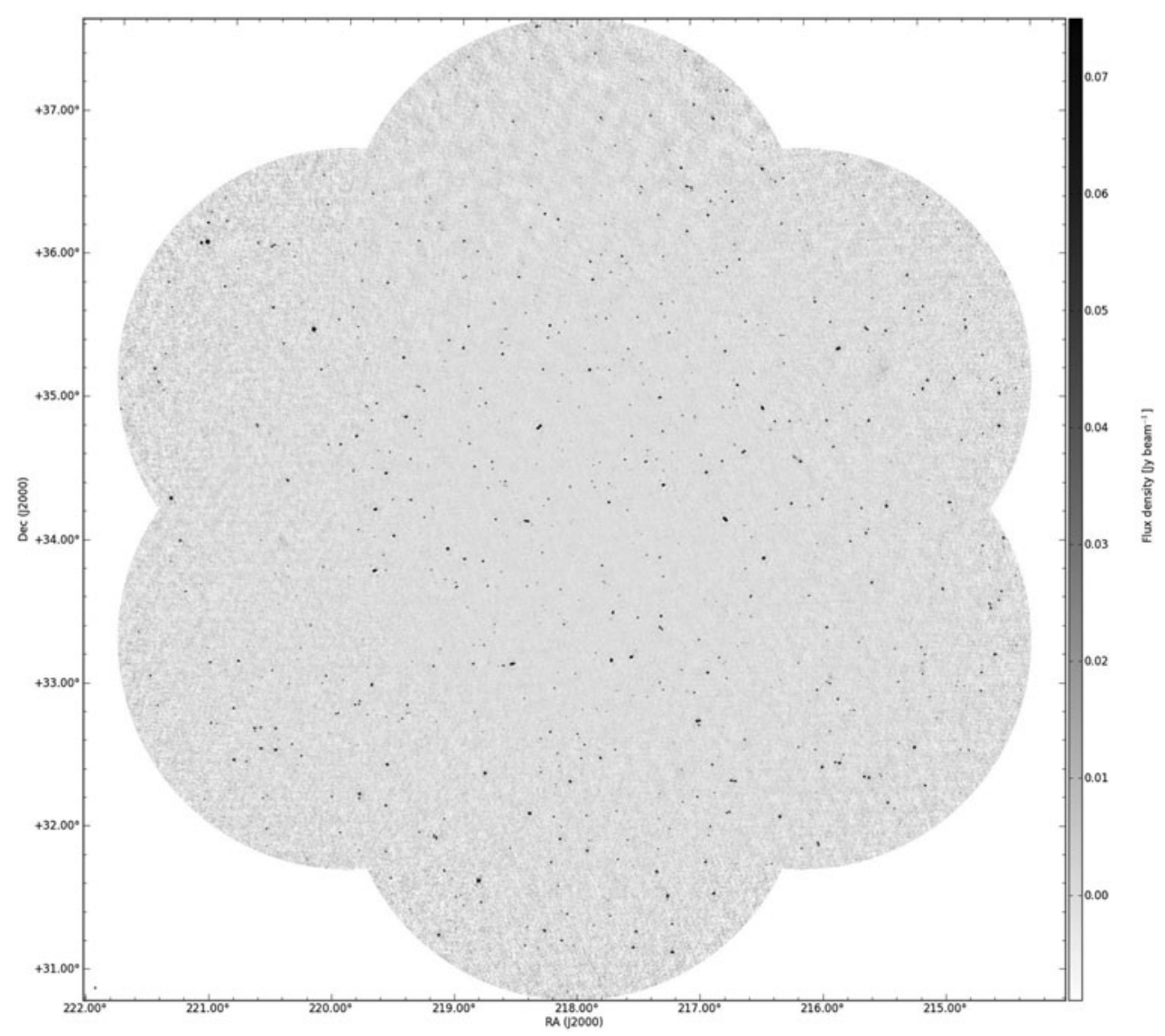

Figure 4. The GMRT Boötes $153 \mathrm{MHz}$ mosaic covering 30 square degrees. The greyscale shows the flux density from $-3 \sigma$ to $25 \sigma$ where $\sigma=3.0 \mathrm{mJy} \mathrm{beam}^{-1}$ is the average $\mathrm{rms}$ across the entire mosaic.

(NDWFS; Jannuzi et. el. 1999) and covers $\sim 9 \operatorname{deg}^{2}$ in the optical and near infra-red $B_{W}$, $R, I$ and $K$ bands. There is a wealth of additional complementary data available for this field, including X-ray (Murray et al. 2005, Kenter et al. 2005), UV (GALEX; Martin et al. 2003), and mid infrared (Eisenhardt et al. 2004, Martin et al. 2003). The region has also been surveyed at radio wavelengths with the WSRT at both $1.4 \mathrm{GHz}$ (de Vries et al. 2002) and $325 \mathrm{MHz}$ (Croft et al. 2008), and the VLA at $1.4 \mathrm{GHz}$ (Higdon et al. 2005). Recently, the AGN and Galaxy Evolution Survey (AGES) has provided redshifts for 23745 galaxies and AGN across $7.7 \mathrm{deg}^{2}$ of the Boötes field (Kochanek et al. 2012).

Our GMRT observations cover 30 square degrees at a resolution of $25^{\prime \prime}$ (Williams et al. 2013). The resulting mosaic is shown in Fig. 4; the rms noise is $2 \mathrm{mJy}$ beam $^{-1}$ in the centre of the image, rising to $4-5 \mathrm{mJy} \mathrm{beam}^{-1}$ on the edges, with an average of $3 \mathrm{mJy}_{\text {beam }}{ }^{-1}$. Seventy-five per cent of the area has an $\mathrm{rms}<4 \mathrm{mJy} \mathrm{beam}^{-1}$. The extracted source catalogue contains 1289 sources detected at $5 \sigma$, of which 453 are resolved. We have matched these sources to the NDWFS $I$ catalogue using the Likelihood Ratio and are using the multi-wavelength data available to construct SEDs and determine photometric redshifts and host galaxy properties such as stellar mass for the whole GMRT Boötes field sample. Such work will be readily extendible to the larger LOFAR sample soon to be available. 


\section{Prospects}

LOFAR has been producing excellent quality interferometric data for a number of years now. The commissioning period is over and Cycle 1 observations started in November 2013. During the commissioning period, a number of crucial challenges were overcome relating to the calibration and imaging of low frequency aperture array interferometric data, including handling RFI, beam calibration, bright source removal and wide-field imaging.

LOFAR Surveys have already started imaging a handful of deep fields towards the surveys Tier-1 depth. The combination of the LOFAR surveys data and other ancillary multi-wavelength data available in many of the extra-galactic deep fields will make LOFAR a great tool for studies of AGN, distant clusters and galaxies.

\section{Acknowledgements}

LOFAR, the Low Frequency Array, designed and constructed by ASTRON, has facilities in several countires, that are owned by various parties (each with their own funding sources), and that are collectively operated by the International LOFAR Telescope (ILT) foundation under a joint scientific policy. The results presented here are the result of significant work and effort by a large number of people. We acknowledge the engineers who have designed and built the array, as well as the number of commissioners who have contributed to the progress of imaging with LOFAR to date.

\section{References}

Asgekar, A., Oonk, J. B. R., Yatawatta, S., et al. 2013, A\&A, 551, L11

Best, P. N. \& Heckman, T. M. 2012, MNRAS, 421, 1569

Bhatnagar, S., Cornwell, T. J., Golap, K., \& Uson, J. M. 2008, A\& A, 487, 419

Croft, S., van Breugel, W., Brown, M. J. I., et al. 2008, AJ, 135, 1793

de Gasperin, F., Orrú, E., Murgia, M., et al. 2012, A\&A, 547, A56

de Vries, W. H., Morganti, R., Röttgering, H. J. A., et al. 2002, AJ, 123, 1784

Eisenhardt, P. R., Stern, D., Brodwin, M., et al. 2004, ApJS, 154, 48

Higdon, J. L., Higdon, S. J. U., Weedman, D. W., et al. 2005, ApJ, 626, 58

Intema, H. T., van der Tol, S., Cotton, W. D., et al. 2009, A\&A, 501, 1185

Jannuzi, B. T. \& Dey, A., NDWFS Team. 1999, in BAAS, Vol. 31, 1392

Janssen, R. M. J., Röttgering, H. J. A., Best, P. N., \& Brinchmann, J. 2012, A $\&$ A, 541, A62

Kenter, A., Murray, S. S., Forman, W. R., et al. 2005, ApJS, 161, 9

Kochanek, C. S., Eisenstein, D. J., Cool, R. J., et al. 2012, ApJS, 200, 8

Martin, C., Barlow, T., Barnhart, W., et al. 2003, SPIE, Vol. 4854, 336-350

Murray, S. S., Kenter, A., Forman, W. R., et al. 2005, ApJS, 161, 1

Offringa, A. R., de Bruyn, A. G., Biehl, M., et al. 2010, MNRAS, 405, 155

Röttgering, H. J. A. 2010, ISKAF2010 Science Meeting

Tasse, C., van der Tol, S., van Zwieten, et al., S. 2013, A\&3A, 553, A105

van der Tol, S., Jeffs, B. D., \& van der Veen, A.-J. . 2007, IEEE Trans. on Signal Processing, 55,4497

van Haarlem, M. P., Wise, M. W., Gunst, A. W., et al. 2013, A\&ऽA, 556, A2

van Weeren, R. J., Röttgering, H. J. A., Rafferty, D. A., et al. 2012, A\&گA, 543, A43

Williams, W. L., Intema, H. T., \& Röttgering, H. J. A. 2013, A\&A, 549, A55

Yatawatta, S., de Bruyn, A. G., Brentjens, M. A., et al. 2013, A 6 A, 550, A136 